\title{
CARACTERIZAÇÃO DOS PERFIS DE CRESCIMENTO CELULAR DE MEYEROZYMA CARIBBICA EM MEIOS DE CULTURA CONTENDO GLICOSE, XILOSE E CELOBIOSE
}

\author{
V. TADIOTO ${ }^{1 *}$, L. M. MILANI ${ }^{1}$, E. T. BARRILLI ${ }^{1}$, H. TREICHEL ${ }^{2}$ e S. L. ALVES JR ${ }^{1}$ \\ ${ }^{1}$ Engenharia Ambiental e Sanitária, Campus Chapecó, Universidade Federal da Fronteira Sul \\ ${ }^{2}$ Engenharia Ambiental e Sanitária, Campus Erechim, Universidade Federal da Fronteira Sul \\ *E-mail para contato: vivianitadioto@ hotmail.com
}

\begin{abstract}
RESUMO - O etanol tradicional deixa para trás resíduos de bagaço e palha de cana-de-açúcar ricos em açúcares fermentáveis. Esses carboidratos podem, contudo, ser amplamente utilizados numa nova fermentação microbiana (etanol de segunda geração ou 2G). A Saccharomyces cerevisiae, levedura responsável pela produção do etanol de primeira geração, é muito bem adaptada às condições industriais de fermentação alcoólica, porém ela é incapaz de fermentar xilose e celobiose, razão essa que limita a produção de etanol de segunda geração. Levando em conta que a biomassa lignocelulósica do bagaço e da palha de gramíneas é decomposta com o auxílio de diferentes fungos filamentosos e leveduras que, ao contrário da $S$. cerevisiae, são capazes de utilizar esses carboidratos citados, o presente trabalho se propôs a analisar os perfis de crescimento celular de cinco linhagens de Meyerozyma caribbica isoladas de bagaço de milho em decomposição. Todas as linhagens analisadas foram capazes de metabolizar glicose, xilose e celobiose. A glicose foi o açúcar mais rapidamente assimilado pelas células, e os meios com celobiose foram os que proporcionaram maior crescimento celular. Diante dos resultados obtidos, é possível inferir que as leveduras aqui analisadas apresentam potencial para contribuir com a otimização da produção de etanol de segunda geração.
\end{abstract}

\section{INTRODUÇÃO}

O bagaço da cana-de-açúcar é um resíduo proveniente da produção de etanol de primeira geração, obtido a partir da fermentação do caldo dessa gramínea. Esse resíduo representa 150 milhões de toneladas anuais no país; de modo geral a maior parte do bagaço é utilizada para gerar calor e/ou energia elétrica na própria indústria (Stambuk et al., 2008; Silva et al., 2014).

Com o cenário atual de uma crescente demanda de combustíveis no Brasil, nos próximos anos aumentará ainda mais a quantidade desse tipo resíduo. De outro modo, o bagaço e a palha representam uma biomassa lignocelulósica rica em açúcares fermentáveis que podem ser utilizados para a produção do etanol de segunda geração, que é uma alternativa viável no aumento da produção de etanol, sem a necessidade de haver uma maior área plantada dessa gramínea (Zanin et al., 2000; Stambuk et al., 2008; Soccol et al., 2010). 
A levedura Saccharomyces cerevisiae faz a fermentação do caldo da cana-de-açúcar para a produção do etanol de primeira geração. Essa levedura é muito bem adaptada às condições industriais e aos estresses provocados por estes procedimentos. Entretanto, ela é incapaz de metabolizar significativa parcela dos carboidratos resultantes da hidrólise da celulose e da hemicelulose que compõem a biomassa lignocelulósica das gramíneas. Entre esses açúcares não metabolizados por $S$. cerevisiae, estão a celobiose e a xilose. Desse modo, alguns pesquisadores veem a engenharia genética dessa levedura como opção para a viabilização da produção de etanol 2G (Stambuk et al., 2008; Lee et al., 2013). Por outro lado, há trabalhos recentes que demonstram a possibilidade do emprego de outras espécies de leveduras nas dornas de fermentação como alternativa à engenharia genética de $S$. cerevisiae (Reis et al., 2014; Wang et al., 2016).

A espécie Meyerozyma caribbica (Vaughan-Martini et al., 2005; Kurtzman e Suzuki, 2010) apresenta-se como uma das alternativas à $S$. cerevisiae, haja vista já ter sido isolada de matéria vegetal em decomposição como bagaço de agave-azul, casca de banana, sorgo e sementes de cacau (N'guessan et al., 2011; Papalexandratou e De Vuyst, 2011; Saucedo-Luna et al., 2011; Hande et al., 2013). Contudo, há ainda pouca informação acerca do metabolismo dessa levedura diante dos carboidratos encontrados em hidrolisados de biomassa lignocelulósica, especialmente celobiose. Nessas circunstâncias, este trabalho se propôs a caracterizar os perfis de crescimento celular da levedura Meyerozyma caribbica, isolada de bagaço de milho em decomposição, em meios contendo três dos principais açúcares oriundos da hidrólise da celulose e da hemicelulose: glicose, celobiose e xilose.

\section{MATERIAIS E MÉTODOS}

\subsection{Isolamento e Identificação das Leveduras}

As leveduras foram isoladas conforme protocolo de Cadete et al. (2009) a partir de amostras de bagaço e palha de milho em decomposição, obtidas nas áreas experimentais do Campus Chapecó da Universidade Federal da Fronteira Sul. As amostras foram coletadas em tubos Falcon de $50 \mathrm{~mL}$ estéreis. Aproximadamente $1 \mathrm{~g}$ foi inoculada em $20 \mathrm{~mL}$ de meio sintético mínimo contendo $0,67 \%$ de base nitrogenada, $0,02 \%$ de cloranfenicol e $1 \%$ de xilose. Foram preparados quatro frascos erlernmeyers e incubados a $25^{\circ} \mathrm{C}$ por $3-10$ dias, sob agitação periódica de $145 \mathrm{rpm}$. Quando se detectou crescimento celular por turbidez, uma alçada de células foi estriada por esgotamento em placas de Petri, essas contendo os mesmos meios supracitados, alem da adição de $2 \%$ de ágar. As placas foram incubadas a uma temperatura de $28^{\circ} \mathrm{C}$, e as leveduras foram isoladas pela morfologia da colônia. As cepas foram nomeadas conforme código pré-estabelecido pela coleção do grupo de pesquisa.

A identificação das leveduras foi realizada no Instituto Biológico do Estado de São Paulo através do sequenciamento da região ITS-5.8S e da subsequente comparação das sequências obtidas com aquelas disponíveis no banco de dados GenBank por meio da ferramenta BLAST (em http://www.ncbi.nlm.nih.gov).

\subsection{Condições de cultivo e crescimento celular}

Dentre as cepas isoladas, cinco linhagens de $M$. caribbica foram escolhidas para avaliação dos perfis de crescimento celular. Inicialmente, as células foram submetidas a um 
pré-inoculo de $48 \mathrm{~h}$ em $10 \mathrm{~mL}$ de meio rico, contendo $1 \%$ de extrato de levedura, $2 \%$ de peptona e $2 \%$ de glicose. Em seguida, foram inoculadas (1/100 do volume final) em frascos erlenmeyers com meio sintético mínimo, contendo $0,67 \%$ de base nitrogenada e $2 \%$ de glicose, xilose ou celobiose como fontes de carbono. Todos os meios foram ajustados em $\mathrm{pH}$ 5,0 . Os frascos foram incubados a $25^{\circ} \mathrm{C}$ sob agitação periódica de $145 \mathrm{rpm}$. Em tempos prédeterminados ao longo do cultivo, retirou-se amostras para a determinação do crescimento celular: uma alíquota de cada uma dessas amostras foi utilizada para determinar a concentração celular através da medida da densidade óptica ( $\left.\mathrm{DO}_{570 \mathrm{~nm}}\right)$ em espectrofotômetro.

\section{Resultados e Discussões}

Com a finalidade de isolar, identificar e caracterizar leveduras com potencial de contribuir com a viabilidade da produção de bioetanol, foram coletadas amostras de bagaço e palha de milho em decomposição e, a partir delas, isoladas 26 linhagens. Dessas 26 linhagens, cinco foram identificadas como pertencentes a espécie Meyerozyma caribbica. Essas cinco linhagens (CHAP-087, CHAP-091, CHAP-096, CHAP-103 e CHAP-108) tiveram seus perfis de crescimento celular em glicose, xilose e celobiose analisados.

As cinco linhagens analisadas foram capazes de metabolizar os três açúcares testados, embora tenham apresentado distintas velocidades de crescimento: enquanto a fase exponencial em meio com glicose teve início em $10 \mathrm{~h}$ de incubação, nos meios com as outras duas fontes de carbono o crescimento logarítmico iniciou apenas após 15-20 h de cultivo (vide Figuras 1, 2 e 3). Esses dados corroboram resultados de outros pesquisadores, que já demonstraram que leveduras tendem a metabolizar a glicose mais rapidamente que outros carboidratos (Santos et al., 2011).

Figura 1 - Perfis de crescimento celular das linhagens CHAP-087, CHAP-091, CHAP-096, CHAP-103 e CHAP-108 em meios mínimos contendo glicose como fonte de carbono.

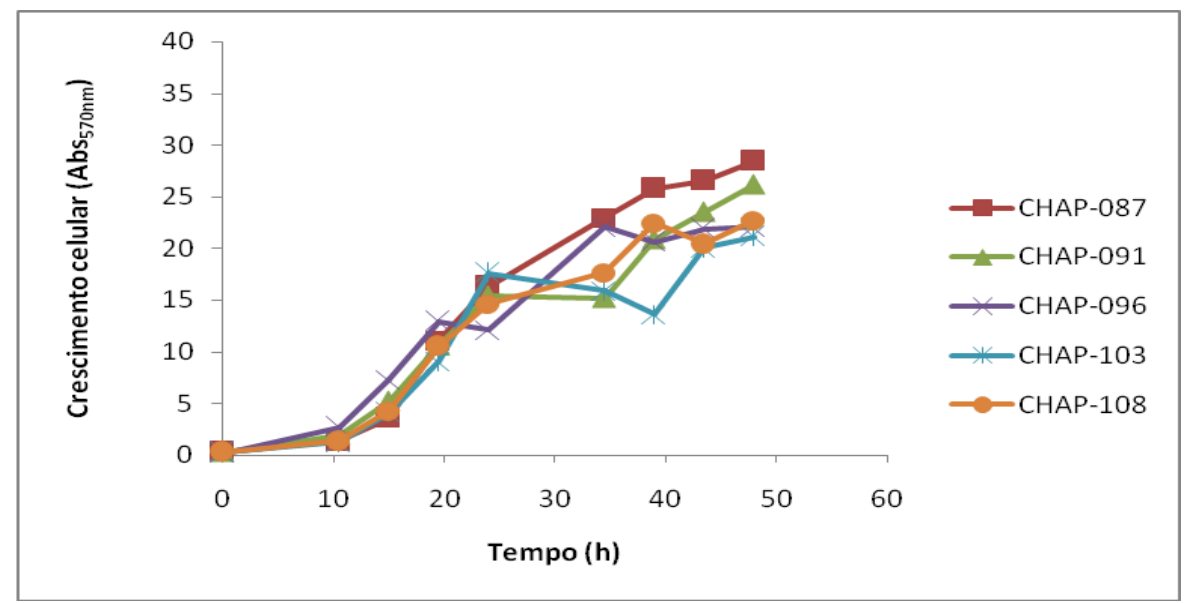


Figura 2 - Perfis de crescimento celular das linhagens CHAP-087, CHAP-091, CHAP-096, CHAP-103 e CHAP-108 em meios mínimos contendo xilose como fonte de carbono.

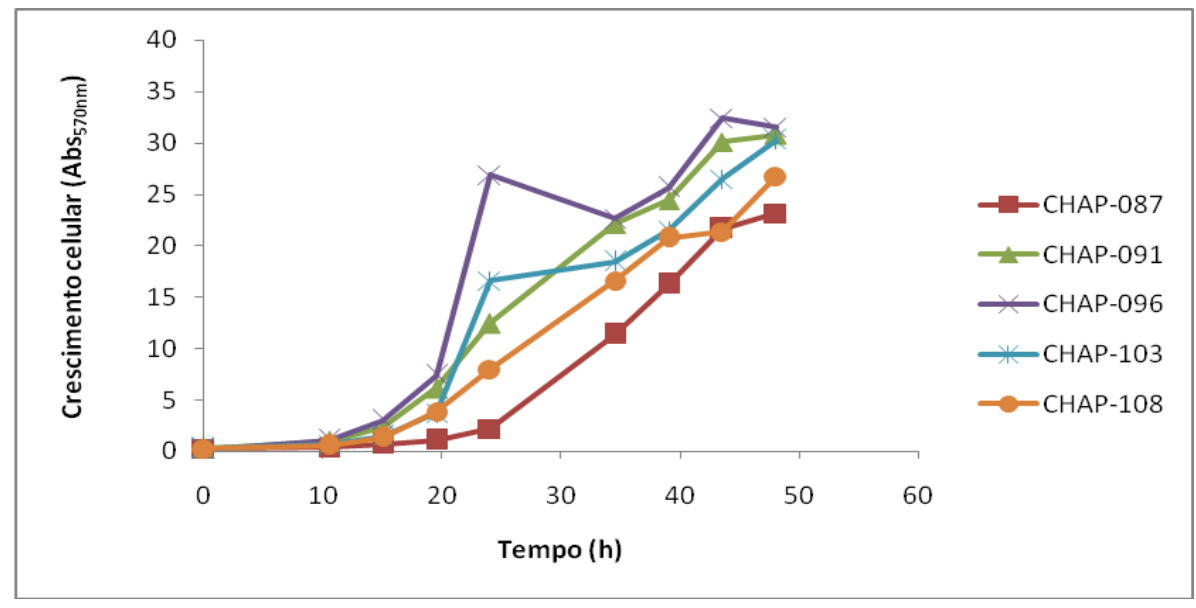

Figura 3 - Perfis de crescimento celular das linhagens CHAP-087, CHAP-091, CHAP-096, CHAP-103 e CHAP-108 em meios mínimos contendo celobiose como fonte de carbono.

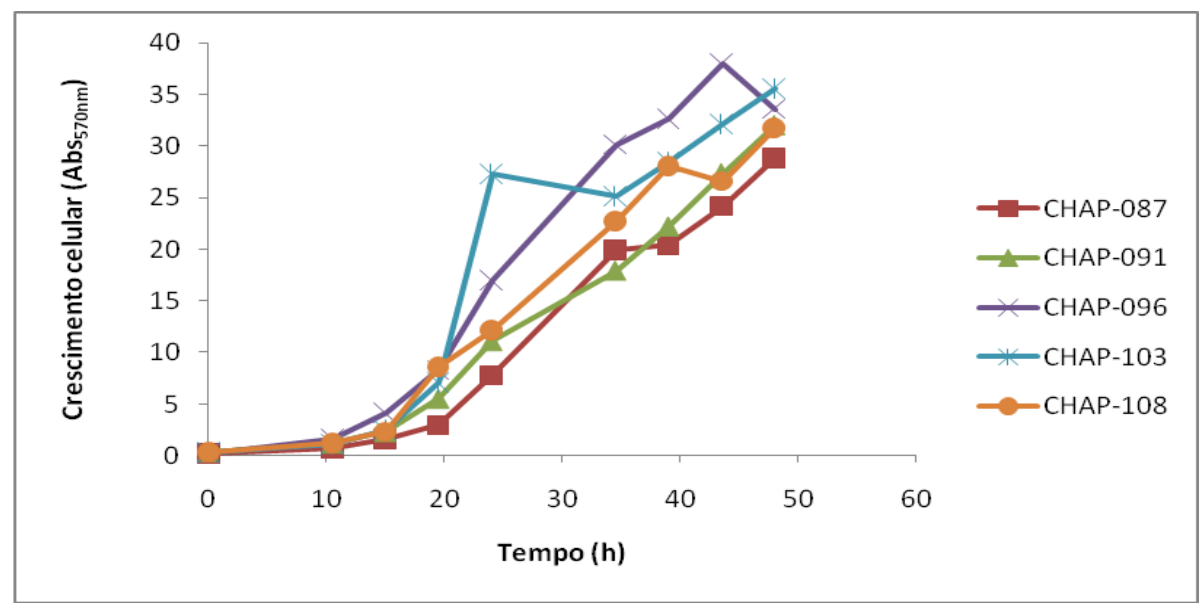

É possível perceber maior crescimento celular das leveduras em meios contendo celobiose em relação ao observado nos meios com os outros dois carboidratos testados. Isso pode ser um indicativo de metabolismo respiratório diante da celobiose e fermentativo diante da glicose e da xilose. De fato, outros autores demonstraram maior rendimento fermentativo quando as células de $M$. caribbica estão metabolizando xilose em vez de celobiose (Hande et al., 2013).

\section{Conclusão}

As cinco linhagens de $M$. caribbica analisadas foram capazes de metabolizar glicose, xilose e celobiose. Tendo em vista que a xilose e a celobiose não são metabolizadas por $S$. 
cerevisiae, as cepas estudadas demonstram potencial para contribuir com a otimização da produção de etanol de segunda geração. Novos estudos poderão verificar a capacidade dessas leveduras de suportar as condições de estresse das dornas de fermentação. Contudo, independentemente dessa capacidade, considerando-se os resultados aqui apresentados, podese inferir que os genes das linhagens de $M$. caribbica, envolvidos no metabolismo de xilose e celobiose, podem vir a ser expressos em $S$. cerevisiae, promovendo às células desta última a capacidade de metabolização desses dois carboidratos.

\section{REFERÊNCIAS}

CADETE, R. M.; SANTOS, R. O.; MELO, M. A.; MOURO, A.; GONÇALVES, D. L.; STAMBUK, B. U.; GOMES, F. C. O.; LACHANCE, M. A.; ROSA, C. A. Spathaspora arborariae sp. nov., a D-xylose-fermenting yeast species isolated from rotting wood in Brazil. FEMS Yeast Res., v. 9, p. 1338-1342, 2009.

HANDE, A.; MAHAJAN, S.; PRABHUNE, A. Evaluation of ethanol production by a new isolate of yeast during fermentation in synthetic medium and sugarcane bagasse hemicellulosic hydrolysate. Ann. Microbiol., v. 63, p 63-70, 2013.

KURTZMAN, C. P.; SUZUKI, M. Phylogenetic analysis of ascomycete yeasts that form coenzyme Q-9 and the proposal of the new genera Babjeviella, Meyerozyma, Millerozyma, Priceomyces, and Scheffersomyces. Mycoscience, v. 51, p. 2-14, 2010.

LEE, W. H.; Nan, H.; Kim, H. J.; Jin, Y. S. Simultaneous saccharification and fermentation by engineered Saccharomyces cerevisiae without supplementing extracellular Bglucosidase. J. Biotechnol., v. 167, p. 316-322, 2013.

N'GUESSAN, K.F.; BROU, K.; JACQUES, N.; CASAREGOLA, S.; DJE, K.M. Identification of yeasts during alcoholic fermentation of tchapalo, a traditional sorghum beer from Côte d'Ivoire. Antonie Van Leeuwenhoek, v. 99, p. 855-64, 2011.

PAPALEXANDRATOU, Z.; DE VUYST, L. Assessment of the yeast species composition of cocoa bean fermentations in different cocoa-producing regions using denaturing gradient gel electrophoresis. FEMS Yeast Res., v. 11, p. 564-574, 2011.

REIS, A. L. S.; DE SOUZA, R. F. R.; TORRES, R. R. N. B.; LEITE, F. C. B.; PAIVA, P. M. G.; VIDAL, E. E.; MORAIS, M. A. Jr. Oxygen-limited cellobiose fermentation and the characterization of the cellobiase of an industrial Dekkera/Brettanomyces bruxellensis strain. SpringerPlus, v. 3, p. 38, 2014.

SANTOS, R. O.; CADETE, R. M.; BADOTTI, F.; MOURO, A.; WALHEIM, D. O.; GOMES, F. C. O.; LACHANCE, M. A.; ROSA, C. A. Candida queiroziae sp. nov., a cellobiose-fermenting yeast species isolated from rotting wood in Atlantic Rain Forest. Antonie Van Leeuwenhoek, v. 99, p. 635-642, 2011.

SILVA, L. F.; TACIRO, M. K.; RAICHER, G.; PICOLLI, R. A.; MENDONÇA, T. T.; LOPES, M. S.; GOMEZ, J. G. Perspectives on the production of polyhydroxyalkanoates 
in biorefineries associated with the production of sugar and ethanol. Int. J. Biol. Macromol., v. 71, p. 2-7, 2014.

SOCCOL, C. R.; VANDENBERGHE, L. P.; MEDEIROS, A. B.; KARP, S. G.; BUCKERIDGE, M.; RAMOS, L. P.; PITARELO, A. P.; FERREIRA-LEITÃO, V.; GOTTSCHALK, L. M.; FERRARA, M. A.; DA SILVA BOM, E. P.; DE MORAES, L. M.; ARAÚJO, J. A.; TORRES, F. A. Bioethanol from lignocelluloses: Status and perspectives in Brazil. Bioresource Technology, v. 101, p. 4820-4825, 2010.

SAUCEDO-LUNA, J.; CASTRO-MONTOYA, A.J.; MARTINEZ-PACHECO, M.M.; SOSA-AGUIRRE, C.R.; CAMPOS-GARCIA, J. Efficient chemical and enzymatic saccharification of the lignocellulosic residue from Agave tequilana bagasse to produce ethanol by Pichia caribbica. J. Ind. Microbiol. Biotechnol., v. 38, p. 725-732, 2011.

STAMBUK, B. U.; ELEUTHERIO, E. A.; FLOREZ-PARDO, L. M.; SOUTO-MAIOR, A.; BON, E. S. Brazilian potential for biomass ethanol: challenge of using hexose and pentose cofermenting yeast strains. J. Sci. Ind. Res., v. 67, p. 918-926, 2008.

VAUGHAN-MARTINI, A.; KURTZMAN, C.P.; MEYER, S. A.; O'NEILL, E. B. Two new species in the Pichia guilliermondii clade: Pichia caribbica sp. nov., the ascosporic state of Candida fermentati, and Candida carpophila comb. nov. FEMS Yeast Res., v. 5, p. 463-469, 2005.

WANG. X.; LIU, Z.L.; WEBER, S. A.; ZHANG, X. Two New Native $\beta$-Glucosidases from Clavispora NRRL Y-50464 Confer Its Dual Function as Cellobiose Fermenting Ethanologenic Yeast. PLoS One, v. 11, p. e0151293, 2016.

ZANIN. G. M.; SANTANA C. C.; BOM, E. P.; GIORDANO, R. C.; DE MORAES, F. F.; ANDRIETTA, S. R.; DE CARVALHO NETO, C. C. Brazilian Bioethanol Program. Appl. Biochem. Biotechnol., v. 84-86, p. 1147-1161, 2000. 